\title{
Cerebral Autoregulation: The Concept the Legend the Promise
}

\author{
Christos Lazaridis ${ }^{*}$ (1) \\ (c) 2021 Springer Science+Business Media, LLC, part of Springer Nature and Neurocritical Care Society
}

Pressure-flow autoregulation refers to the physiologic phenomenon of the maintenance of steady flow within a range of arterial blood pressure (ABP). It is one of the mechanisms that collectively contribute to the regulation of cerebral blood flow (CBF). Brain tissue has high metabolic demand and limited substrate storage capacity; for this, efficient and precise regulation of CBF is required. The other mechanisms involved describe relationships between $\mathrm{CBF}$ and the partial pressures of $\mathrm{CO}_{2}$ and $\mathrm{O}_{2}$, as well as perivascular $\mathrm{pH}$ (chemical vasoreactivity), flowmetabolism coupling, and autonomic neurogenic control of the vasculature. Truth be told, there is much more that we do not know about these mechanisms and how they overlap, compared with what we would call "facts." Nevertheless, the observation about vascular pressure reactivity was made over a century ago. Bayliss [1] reported on myogenic vasomotor tone change in response to blood pressure fluctuations. However, it was not until 1959 and the work of Lassen that gave us the textbook static cerebral pressure autoregulation graph. Lassen constructed a plot of average ABP and total CBF from seven studies involving 11 different patient groups exhibiting variable $A B P$ levels due to either pharmacologic or pathologic reasons [2]. The plot is classically depicted as having a plateau section of unvarying CBF between upper and lower inflection points beyond which CBF is linearly and passively related to ABP. It turns out that as neat as the graph appears, it is likely an oversimplification, may be a function of the experimental conditions in which it is assessed, and its definitive efficacy remains

\footnotetext{
*Correspondence: lazaridis@uchicago.edu Departments of Neurology and Neurosurgery, University of Chicago Medical Center, 5841 S. Maryland Ave. MC 2030, Chicago, IL 60637, USA
}

This article is related to the original article available at https://doi. org/10.1007/s12028-020-01185-x.

\section{Springer}

uncertain. Other controversies include the role of the speed and direction in ABP change (there is evidence of hysteresis, i.e., the brain defends more effectively against acute hypertension than hypotension); the anatomical site of regulation, as in pial arterioles vs. larger intracranial arteries; myogenic vs. neurogenic vs. local metabolic modulation; treating as separate entities chemical and pressure reactivity [3].

Despite incomplete understanding, the relationship between ABP fluctuations (spontaneous or induced) and intracranial pressure (ICP) is clinically commonly observed and exploited in order to identify an optimal combination between mean arterial pressure (MAP) and ICP (and derivative cerebral perfusion pressure; $\mathrm{CPP})$. The so-called MAP challenge is incorporated in recent targeted protocols for the management of severe traumatic brain injury (TBI) such as the protocol for the BOOST-3 trial and the SIBICC algorithm [4]. An intuitive idea arises, that of a method of continuous bedside monitoring of cerebrovascular pressure reactivity and/or pressure-flow autoregulation that would not be dependent on artificial manipulation of ABP. This idea was given form by Czosnyka et al. by deriving the pressure reactivity index (PRx); a moving correlation coefficient from 30 consecutive 10-s averages of ICP and ABP waveforms [5]. Since then, the Cambridge group (and disciples) has generated a large body of literature including new indices, validation studies, and observational outcome data in various acute brain injury (ABI) pathologies. It is useful to offer an epigrammatic account of these accomplishments: PRx has been shown to independently correlate with clinical outcome after TBI [6]; change of PRx from zero or negative to positive identifies Lassen's lower inflection point under experimental conditions [7]; PRx plotted against CPP shows a U-shaped curve, whose minimum theoretically corresponds to the plateau section of the curve. This value was termed the "optimal 
$\mathrm{CPP}$ " $\left(\mathrm{CPP}_{\mathrm{OPT}}\right)$. Pressures lower and higher than $\mathrm{CPP}_{\mathrm{OPT}}$ have been associated with worse outcomes [8]; intracranial hypertension in the presence of pressure passivity is related to worse outcomes independently from the absolute ICP burden, which motivates the idea of individualizing the ICP threshold [9].

In summary, what we currently have is on one side fragmentary physiologic understanding and on the other excitement with the potential for clinically meaningful continuous autoregulation monitoring. Within this context, a timely effort was undertaken by 25 experts who via a Delphi process reviewed the available methodological and clinical literature aiming to consensus for use in clinical practice, in conjunction with arriving to a common research agenda [10]. In order to assess and monitor cerebral autoregulation (CA) in the absence of direct CBF monitoring, one has to rely on $\mathrm{CBF}$ surrogate measures. The methods that the Leuven group reviewed can be categorized by employing one of the following surrogates: transcranial Doppler flow velocity (transfer function analysis, ARI, Mx), ICP (PRx, L-PRx, LAx), brain tissue oxygenation (ORx, TOx, THx), and microdialysis derived glutamate. Two additional methods employ direct local CBF measurement (Lx, CBFx). These metrics express time-synchronized and averaged moving correlation coefficients between ABP (or CPP) changes and the corresponding surrogate. Note that these surrogates maybe physiologically related yet are distinct, and depending on the pathophysiologic conditions divergent; ICP is a surrogate of cerebral blood volume, it follows that resultant indices are more precisely markers of cerebrovascular pressure reactivity (e.g., PRx). Tissue oxygenation and metabolism are not solely determined by CBF. In fact, they may be dissociated (or uncoupled) from CBF such as in cases of barriers to oxygen diffusion or primary mitochondrial dysfunction [11]. Another important point relates to the fact that derived indices are noisy and require time-averaging over hours; primary signals have to be filtered to remove high-frequency transients and oscillations, in order to sample slow vasomotor waves. This literature has significant limitations including lack of a gold standard for clinical measurements, few validation studies under experimental conditions, no prospective investigations, and mostly single-center data. It is then not surprising that the group reached no consensus on any of the following substantive issues: the manner how information on CA status should be used in clinical practice; sufficient accuracy, reproducibility, validity of any CA assessment method; and crucially no consensus on the safety of implementing CA status in clinical practice. The last point should make clear that these methods remain experimental and their prospective interventional application should be undertaken under carefully executed clinical trial protocols and after informed consent. Such a trial is the recently completed COGiTATE phase II investigation on the feasibility and safety of monitoring and targeting $\mathrm{CPP}_{\mathrm{OPT}}$ in TBI [12].

I recently argued that physiologic thresholds for treatment, such as for ICP/CPP, could be conceptualized as constructs that involve clinical problem-solving heuristics $[13,14]$. A clinical heuristic is a problem-solving approach employing a practical method that is not guaranteed to be perfect or true, but which may nevertheless be sufficient for reaching a certain clinically relevant goal. Analogously, the indices discussed above including PRx and $\mathrm{CPP}_{\mathrm{OPT}}$ are statistical constructs that appear to have value in patient risk stratification, and in epidemiologic association with poor outcome [15]. For a heuristic to be successful, important merits are ease of application, reproducibility, and the ability to provide concrete decision-making in complex situations. For $\mathrm{CPP}_{\mathrm{OPT}}$, some of these features we expect to learn from COGiTATE. Eventually, if autoregulation monitoring and targeting is to inform $\mathrm{ABI}$ treatment allowing precision individualized approaches will depend on (a) obtaining tissue-outcome data in terms of contributing to alleviating cerebral dysoxia and energy metabolic crisis, and (b) examining how autoregulation data can be incorporated into treatment protocols that shift the benefit-risk of interventions toward improved patient outcomes.

Source of Support

There is no funding in relation to the current manuscript.

Conflict of interest

The author declares that he has no conflict of interest.

\section{Publisher's Note}

Springer Nature remains neutral with regard to jurisdictional claims in published maps and institutional affiliations.

Received: 28 December 2020 Accepted: 31 December 2020

Published online: 25 January 2021

\section{References}

1. Bayliss WM. On the local reactions of the arterial wall to changes of internal pressure. J Physiol. 1902;28(3):220-31.

2. Lassen NA. Cerebral blood flow and oxygen consumption in man. Physiol Rev. 1959;39:183-238.

3. Willie CK, Tzeng YC, Fisher JA, Ainslie PN. Integrative regulation of human brain blood flow. J Physiol. 2014;592(5):841-59.

4. Hawryluk GWJ, Aguilera S, Buki A, et al. A management algorithm for patients with intracranial pressure monitoring: the Seattle International Severe Traumatic Brain Injury Consensus Conference (SIBICC). Intensive Care Med. 2019;45(12):1783-94.

5. Czosnyka M, Smielewski P, Kirkpatrick P, Laing RJ, Menon D, Pickard JD. Continuous assessment of the cerebral vasomotor reactivity in head injury. Neurosurgery. 1997;41(1):11-7.

6. Sorrentino E, Diedler J, Kasprowicz M, et al. Critical thresholds for cerebrovascular reactivity after traumatic brain injury. Neurocrit Care. 2012;16(2):258-66. 
7. Brady KM, Lee JK, Kibler KK, Easley RB, Koehler RC, Shaffner DH. Continuous measurement of autoregulation by spontaneous fluctuations in cerebral perfusion pressure: comparison of 3 methods. Stroke. 2008;39(9):2531-7.

8. Aries MJ, Czosnyka M, Budohoski KP, et al. Continuous determination of optimal cerebral perfusion pressure in traumatic brain injury. Crit Care Med. 2012;40(8):2456-63.

9. Lazaridis C, DeSantis SM, Smielewski P, et al. Patient-specific thresholds of intracranial pressure in severe traumatic brain injury. J Neurosurg. 2014;120(4):893-900.

10. Depreitere B, Citerio G, Smith M et al. Cerebrovascular autoregulation monitoring in the management of adult severe traumatic brain injury: a Delphi consensus of clinicians. Neurocrit Care.

11. Lazaridis C. Cerebral oxidative metabolism failure in traumatic brain injury: "brain shock." J Crit Care. 2017;37:230-3.
12. Beqiri E, Smielewski P, Robba C, et al. Feasibility of individualized severe traumatic brain injury management using an automated assessment of optimal cerebral perfusion pressure: the COGITATE phase II study protocol. BMJ Open. 2019;9(9):e030727.

13. Lazaridis C, Desai M, Damoulakis G, Zeiler FA. Intracranial Pressure Threshold Heuristics in Traumatic Brain Injury: One, None, Many! Neurocrit Care. 2020;32(3):672-6.

14. Lazaridis C, Goldenberg FD. Intracranial pressure in traumatic brain injury: from thresholds to heuristics. Crit Care Med. 2020;48(8):1210-3.

15. Akhondi-Asl A, Vonberg FW, Au CC, Tasker RC. Meaning of intracranial pressure-to-blood pressure fisher-transformed Pearson correlationderived optimal cerebral perfusion pressure: testing empiric utility in a mechanistic model. Crit Care Med. 2018;46(12):e1160-6. 